\title{
Grade 9 Learners' Errors And Misconceptions In Addition Of Fractions
}

\author{
Zwelithini Bongani Dhlamini \\ Israel Kibirige \\ University of Limpopo, School of Education. Department of Mathematics, \\ Science and Technology Education (DMSTE), South Africa \\ Corresponding authors Israel.Kibirige@ul.ac.za
}

\section{Doi:10.5901/mjss.2014.v5n8p236}

\begin{abstract}
This article presents findings of a study on the addition of fractions in South Africa, Gauteng Province. Addition of fractions and algebraic manipulation are key concepts for high school mathematics and it is vital that learners grasp all algorithms related to fractions. This study examined the addition of fractions and the study revealed that a majority of grade 9 learners correctly add fractions if they were numerical but did not perform well when they were algebraic. Furthermore, the findings revealed that when adding algebraic fractions, learners produce errors and reveal misconceptions as a result of short cuts that they perceive as easier methods of adding the fractions instead of following the apparent long and cumbersome algorithmic correct method of adding fractions.
\end{abstract}

Keywords: Learners' errors, misconceptions, addition of fractions, algebraic and algorithms

\section{Introduction}

A concern has been raised that learners perform badly in algebra questions that involve fractions and that knowledge of fractions is crucial for success in algebra (Hart, 1981; Gabriel, et al., 2013; Booth, Newton and Twiss-Garrity, 2014). Norton and Boyce (2013) and Siegler, Thompson and Schneider (2011) argue that fractions are most difficult to teach, most cognitively challenging and most essential for advanced mathematics. Duzenli-Gokalp and Sharma (2010) argues that the difficulty that learners face when adding fractions is as a result of improper connections of everyday examples and mathematical problems on the addition of fractions. In our moderation of learners' work at both the Senior (Grades 79) and Further Education and Training (FET) phases (Grades 10-12), respectively, we have identified errors that are traced back to primary school and misconceptions that learners demonstrate during formal assessments. These errors and misconceptions spurred this study to identify them, and explore what they mean to the learning of fractions. Such an enquiry could contribute to the effective learning of addition of fractions. In this study, constructivism was used as theoretical framework and a lens to view errors and misconceptions that learners displayed when adding fractions. Learners were given tasks and interviewed to elaborate errors and misconceptions

\section{Purpose of the Study}

The purpose of this study was to investigate learners' ideas when adding algebraic fractions. Focus of this inquiry was on errors and misconceptions that learners exhibited when adding simple fractions, and fractions of algebraic numerators and denominators respectively. The first objective of this study was to investigate learners' errors and misconceptions using a mathematics task on algebraic fractions, and real-world context. The second objective was to identify learners' understanding of the addition of algebraic fractions through the use of semi-structured interviews. The study used the following research questions.

1. What errors are made when learners add fractions where numerators are variables?

2. What errors are made when learners add fractions where denominators are variables?

3. What do learners understand about adding algebraic fractions: a) When a context is given?; b) When denominators are the same?; and c) When denominators are not the same? 


\section{What are the Errors and Misconceptions?}

There are discursive mistakes and challenges that learners display in their responses to Mathematics tasks. Luneta and Makonye (2010) pointed out that errors and misconceptions may relate but they are different and they identified two types of errors: systematic and unsystematic errors. According to Lukhele, Murray and Olivier (1999) unsystematic errors are exhibited without the intention of learners; learners may not repeat such errors and learners can correct them independently. According to Watanabe (1991) some learners use short cuts to solve mathematical problems which result in errors. Systematic errors may be repeated, systematically constructed or reconstructed over a period of time due to grasp of incorrect conceptions of solving a particular problem (Idris, 2011). Nesher (1987) described misconceptions as a display of an already acquired system of concepts and algorithms that have been wrongly applied.

\section{Theoretical Framework and Literature Review}

The study used constructivism as a theory and lens to view errors and misconceptions that learners displayed when adding fractions. Bruner (1996) defined constructivism as the process whereby learners actively construct their understanding and knowledge of the world, through experiencing things and reflecting on those experiences. Bruner further states that when learners encounter new things, they reconcile them with their previous ideas and experiences where changes are made or the rejection of new ideas if found to be irrelevant with the help of a knowledgeable instructor whose role is to mediate the learner's construction of knowledge.

According to Vygotsky (1978), constructivism refers to how learners socially construct knowledge linguistically through interacting with signs and symbols. Vygotsky introduced two important concepts to constructivism: the first one is the Zone of Proximal Development (ZPD). Vygotsky described the ZPD as the distance between two placeholders, potential development and actual development. Potential development is the learners' ability to solve mathematical problems with the help of a more knowledgeable peer or adult and actual development is the complete developmental cycle which is shown when learners solve mathematical problems independently (Vygotsky, 1978). The second concept that Vygotsky raised was scaffolding, which refers to the help given to learners by a teacher or more knowledgeable peer at the beginning of a learning activity and is suddenly withdrawn to allow the learner to build mental structures in collaboration with signs and symbols (Vygotsky, 1978). In the context of this study, we viewed errors and misconceptions that learners performed independently when responding to the task on adding fractions.

Watts and Bentley (1991) argue that constructivism is a theory brought about by perception, language, memory, and the ability to enquire beyond given information. Olivier (1989) describes constructivism as the resultant knowledge of interaction between learners, learning experiences and previous knowledge. The quality of existing knowledge drives learning in constructivism. Ausubel (1985) points out that constructivism refer to construction of meaning through hypothesis generation, testing and generalizations.

Gabriel et al. (2013) pointed out that the wrong conceptions resulted in learners facing difficulty in working with fractions because of the multifaceted nature of fractions which is, ratio, operator, quotient and measure. In the context of this study these are: ratio means interpreting fractions as ratio of boys to girls, 2:7, meaning $\frac{2}{7} \mathrm{f}$ the group; operator $\frac{2}{7}$ means 2 divide by 7 or $\frac{1}{7}$ multiplied by 2; quotient is the result of division; measure describes fractions as numbers and intervals. Another important notion revealed by Gabriel et al. (2013) is the 'part- whole' nature of fractions used in sharing. For an example; one litre of cold drink shared equally amongst 7 learners, here the litre need to be divided into 7 equal parts. The notion of the operator for division may be used; $1000 \mathrm{ml}$ divided by 7 and each learner gets $142.85 \mathrm{ml}$. In this same problem, one can pour water in a litre and share it equally in 7 glasses. Gabriel et al. (2013) argues that the procedural and conceptual nature of knowledge that fractions demand poses confusion in learners' choice of the correct notion that need to be applied to a specific problem.

Watanabe (1991) reveals that learners perform easy methods which are alternatives and end up making errors. He says that it is vital to study these alternatives and correct them with learners as they will become learners' previous knowledge. Watanabe called these alternative methods 'short-cuts' and he pointed out that the short-cuts are easier attempts by learners to solve the problem. Lukhele et al. (1999) argue that teaching fractions involves showing fractions as tools for equivalence and sharing. When dealing with fractions the notion of equivalence is used to share wholes equally. A short-cut, as described by Watanabe, will not constitute a correct tool of solving the problem. Hecht and Vagi (2012) attribute this deficiency of adding fractions to lack of conceptual knowledge which is knowledge that governs this domain. A study by Hallett, et al. (2012) revealed that learners often have the following imbalances in procedural and conceptual knowledge of fractions; more conceptual, more procedural, equally good on both, or equally poor on both. 
These discrepancies are as a result of prior knowledge of fractions.

Learners often applied algorithms from other procedures in new concepts, for example, learners presumed that fractions with big whole numbers were bigger fractions because they are employing incorrectly the algorithm of whole numbers (Hart, 1981; Lukhele et al., 1999). Siegler, Fazio, Bailey and Zhou (2013) explain that fractions are difficult for many learners because learners assume that algorithms, procedures and properties of whole numbers are also properties of all other numbers. Bush and Karp (2013) explain that such conceptions come as a result of learners not paying special attention to procedures of fractions such as; comparing and ordering which is the identification of different forms of numbers, fractions are rational numbers and do not hold place values as whole numbers. From 1 and 2 there are two whole numbers yet there are infinite rational numbers. Therefore learners focus on numbers and disregard conceptual knowledge that comes as a result of the amalgamation of concepts (Bush \& Karp, 2013). For an example, learners may add fractions in this manner. $\frac{1}{2}+\frac{1}{2}=\frac{2}{4}$. This is a misconception; the learner took the numbers on the fraction as separate whole numbers, and then added the numerators and the denominators. Valdemoros (1995) argues that great attention must be given to those who use context to add algebraic fractions as real-world contexts bring meaning to the addition of complex algebraic fractions and this is called concrete frameworks.

A study by Ellerton (1991) shows how learners created cognitive links when given notation and terminology by the educator which they were supposed to discuss amongst themselves and add algebraic fractions. The learners were unable to independently use the terminology and notation to add fractions. After what Ellerton called "professional intervention" by teachers, learners were able to use the notation and terminology to add fractions. In constructivism, this intervention is called quality scaffolding (Davydov, 1995).

Olive (1998) carried out a study on how learners used the Lowest Common Denominator (LCD) to add fractions. Learners listed multiples of the two numbers, and used any common denominator to add fractions. Findings of the study revealed that learners correctly reached the required answer provided they simplified their answers.

Another study by Cramer et al. (2008) revealed that representations are useful when used to communicate mathematical approaches, algorithms of adding fractions and to support learners' understanding of concepts for adding fractions and their relationships. A study by Watanabe (2002) showed that representations were meaningful to those who created them; therefore it was critical that teachers allow learners to create their own representations and only guide them to use representations correctly to add fractions, (Siegler et al. 2011). In constructivism these are scaffolds that are essential in the addition of fractions.

Results from a study by Gabriel et al. (2013) revealed that primary school learners master the part-whole and proportion categories of fractions and struggle to understand fraction as numbers which resulted in learners applying procedures that they do not understand.

\section{Methodology}

This was a qualitative study in which we hoped to gain some insights into learners' understanding of algebraic fractions and reasons behind the errors and misconceptions that learners exhibited whilst adding algebraic fractions. Data was collected from learners' responses to a given task and from semi-structured interviews. The qualitative approach was appropriate because the analysis of the learners' responses to the given was used to generate theory.

The task that was given to learners consisted of six questions. Questions 1-4 asked learners to share a chocolate bar between several friends. These questions used the context to work out a problem based on the addition of fractions. Learners were supposed to use the blocks to help them work with algebraic and numerical fractions. The blocks resembled chocolate bars which learners divided, showing exactly what they did to the chocolate bars as an illustration of working with fractions. Question 5 and 6 extended the idea of sharing, out of the everyday context into more abstract fractions with numbers and variables. Question 5 had numerals as denominators and question 6 had numerators being both numerals and variables.

The study was carried out in a public school in Johannesburg Gauteng Province, South Africa. The school served learners in an area that has a high rate of crime. The teachers were multiracial with a majority being female, including the principal. The learners were majority black, a few Indians, and often multilingual. English was the language of instruction, and learners did English as a first language. 


\subsection{Data collection}

A mathematical task based on fractions was given to 40 learners to write independently for 50 minutes under strict supervision such that they did not get help from their peers or educator. We collected all scripts and all 40 learners responded to the task. Learners' scripts were classified, based on the similarity of errors performed in their response to the task. We purposively selected four learners for interviews using a tape recorder for 30 minutes per learner. During the interviews, the learners were asked to respond to tasks containing similar errors and misconceptions that were of interest. Questions that we asked during the interviews sought to find out how learners exhibited certain errors and misconceptions. Below are a few of the questions that we asked during the interviews:

- Explain how you get to this answer.

- Relate what you did in the previous question and think about how you can do the same in this question.

- What are the denominators of these fractions? How can you use them to add these fractions?

- Can you explain further how you solve this problem?

- What do you do to add fractions with the same denominator?

- What do you have to do to solve fractions with different denominators?

\subsection{Data Analysis}

Forty scripts were organised into 4 groups and from those groups, three main themes were generated namely: fractions in a context, fractions with algebraic numerators and fractions with algebraic denominators.

\section{Results}

Learners responses are reported in three themes and statements from four learners are reported verbatim.

\subsection{Fractions in context (Theme 1)}

\subsubsection{Learner A written response}

1. One chocolate bar is shared among 5 friends. How much of the chocolate bar does each friend get? 4 Use the bar below to show your answer.

$20 \div 4=5$ or $5 \times 4=20$

This learner shows the numerical calculations inside the bar provided in the question. This was the learner's response when writing the task and during the interview when we asked the learner to explain a different version was shown in the answer sheet given during the interview. Below are transcripts of response during the interview.

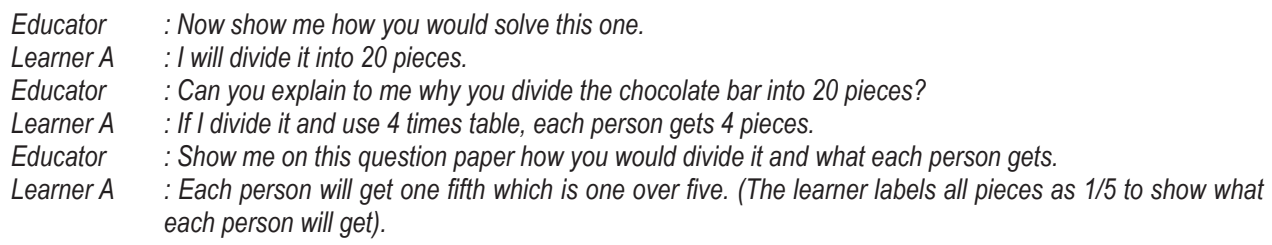

The learners' response during the interview is shown below;

1. One chocolate bar is equally shared among 5 friends. How much of the chocolate bar doe each friend get? $\quad 1 / 5 \quad$ Use the bar below to show your answer.

\begin{tabular}{|l|l|l|l|l|}
\hline $1 / 5$ & $1 / 5$ & $1 / 5$ & $1 / 5$ & $1 / 5$ \\
\hline
\end{tabular}

The learner first said that the chocolate bar will be divided into 20 pieces and then give each friend 4 pieces. After we questioned this, the learner realises that she must apply the algorithm of fractions. Hart (1981) and Siegler et al. (2013) 
states that learners dealing with fractions had the tendency of using whole numbers to solve problems on fractions which result in a misconception which the learner easily corrects after realising how to apply the correct algorithm.

Another question that used a context was question 2 which looked similar to question1, the only difference was that instead of ' 5 friends' question 2 had ' $x$ friends'. One learner's response is shown below:

\subsubsection{Learner $\mathrm{C}$ written response}

2. One chocolate bar is equally shared amongst $x$ friends. How much of the chocolate bar does each friend get? $1 / 4$ Use the bar below to show your answer?

\begin{tabular}{|l|l|l|l|}
\hline $1 / 4$ & $1 / 4$ & $1 / 4$ & $1 / 4$ \\
\hline
\end{tabular}

The learner chose to use a number four instead of working with the variable. The transcript of the interview follows:

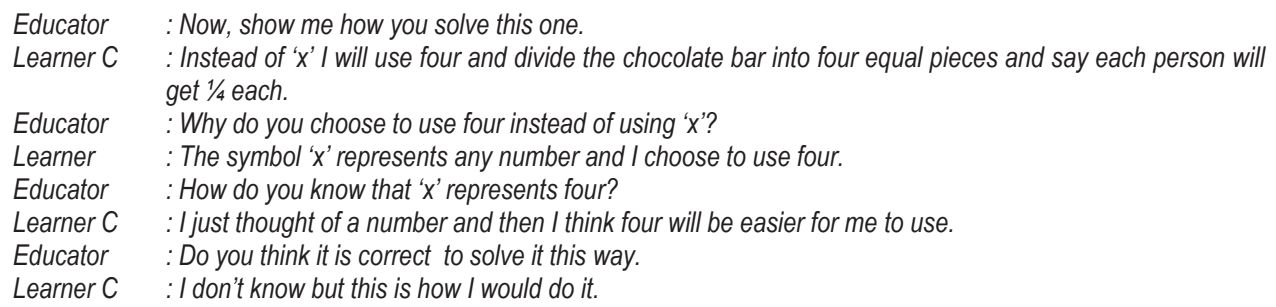

\subsection{Fractions with algebraic numerators (Theme 2)}

A response from learner $B$ is shown below:

\subsubsection{Learner B written response}

3. Simplify the following fractions (show all your working).
a) $\frac{x}{2}+\frac{x}{3}=\frac{x^{2}}{5}$
b) $\frac{x}{3}+\frac{1}{4}=\frac{1 x}{7}$

During the interview, learner B did the same as in the task by adding the denominators and multiplied the numerators. For question $5 \mathrm{~b}$ the learner was questioned and changed the solution.

\subsubsection{Learner B response during the interview}

Simplify the following fractions (show all your working).

$$
\begin{aligned}
& \text { a) } \frac{x}{2}+\frac{x}{3}=\frac{x^{2}}{5} \\
& \text { b) } \frac{x}{3}+\frac{1}{4}=\frac{1 x}{7} \text { or LCD } 12 \frac{4 x}{12}+\frac{3 x}{12}=\frac{7 x}{12}
\end{aligned}
$$

Educator : How can you answer this one?

Learner B : I think I would just add. I will start with the denominators and say three plus four and will give me seven and ' $x$ ' plus one will give me one ' $x$ '.

Educator : Why do you think we must add the denominators and the numerators? What can you say about the fractions?

Learner $B \quad$ : I will have to find the lowest common denominator which is twelve.

Educator : Then solve it.

Learner $B \quad$ : I say how many times does three goes into twelve which is four and say how many times does four goes into twelve which is three. Then four plus three which gives seven ' $x$ ' and the answer is seven ' $x$ ' over twelve. 
6.3 Fractions with algebraic denominators (Theme 3)

\subsubsection{Learner B written response}

Simplify the following fractions (show all your working).
a) $\frac{1}{2}+\frac{3}{x}=\frac{4}{2 x}=2 x$
b) $\frac{1}{x y}+\frac{4}{2 y}=\frac{5}{2 x y^{2}}$

\subsubsection{Learner $B$ transcript of the interview is given below;}

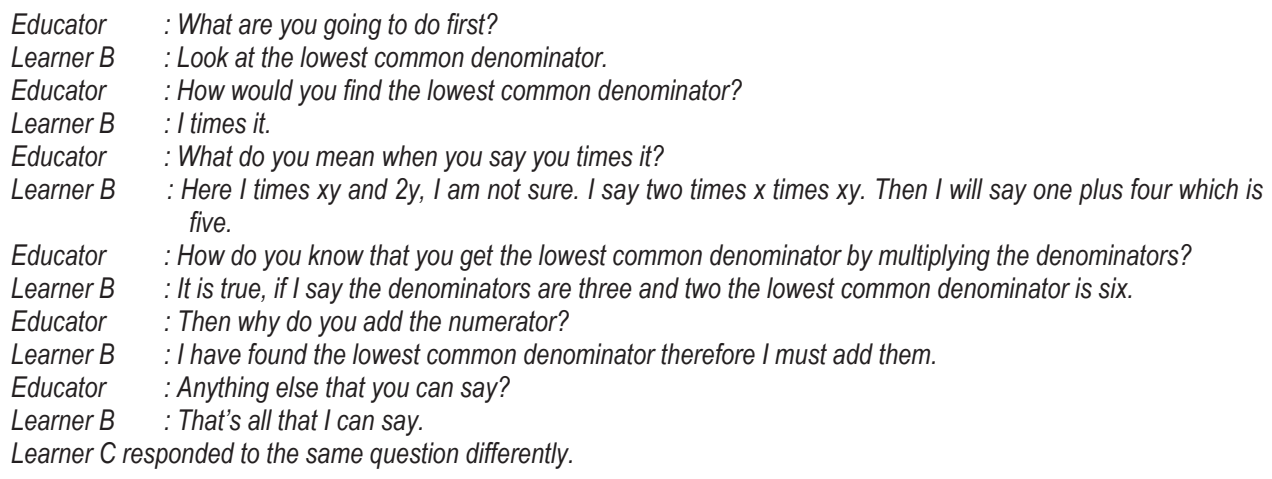

\subsubsection{Learner $\mathrm{C}$ written response}

Simplify the following fractions (show all your working).
a) $\frac{1}{2}+\frac{3}{x}=\frac{x(1)+2(3)}{2 x}=\frac{x+6}{2 x}$
b) $\frac{1}{x y}+\frac{4}{2 x y}=\frac{x y(1)+x y(4)}{2 x y^{2}}$

\subsubsection{Learner $D$ written response}

Simplify the following (show all your working):

c) $\frac{x+x+x+x+x+x}{x}=\frac{x^{6}}{x}$

Educator : How do you think you solve this one?

Learner $D:$ I count the $x$ 's on top and it gives $x$ to the power of six over $x$.

Educator : Let's look at what you have done. Look at the numerator, there is the operation which is added'. Do you think that you only count when you have to add?

Learner $D$ : That is how I simplified it and Sir look, the denominator and the numerator is bigger than the denominator, then I must leave it as it is.

Educator : Explain briefly why you say that you must leave it as it is.

Learner $D:$ We have $x$ to the power of six and the denominator has one $x$ therefore we leave it as it is Sir.

Learner A had a different conception of simplifying the same problem.

\subsubsection{Learner A written response}

Simplify the following fractions (show all you're working).

c) $\frac{x+x+x+x+x+x}{\not}=\frac{x^{5}}{x}$

The learner cancelled the denominator with one $\mathrm{x}$ on the numerator but still kept the cancelled $\mathrm{x}$ on the denominator. The learner applied the algorithm of multiplication instead of applying the algorithm of addition of fractions and this is a misconception. Below is a script from the same learner and transcript from the interview. 


\subsubsection{Learner $\mathrm{C}$ response during the interview}

Simplify the following fractions (show all you're working).

c)

c) $\frac{x+x+x+x+x+x}{x}=\frac{x}{x}+\frac{x}{x}+\frac{x}{x}+\frac{x}{x}+\frac{x}{x}+\frac{x}{x}=1+1+1+1+1+1=6$

The learner here used the algorithm of fraction of using the denominator and simplified correctly. This shows some understanding but it is contrary to what the learner did when she wrote the task individually where the learner did not show understanding of the same problem. The interview transcript showed how the learner responded.

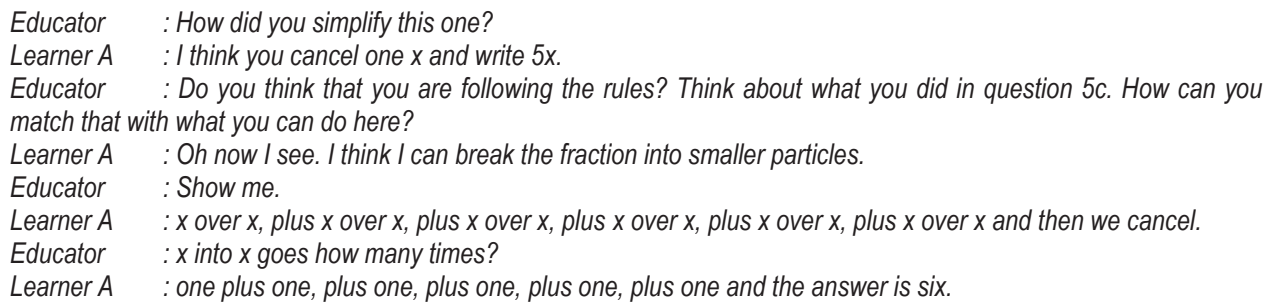

This is an indication that the learner had an idea in solving this problem but the learner did this with the help of the teacher.

\section{Discussion}

The discussion of the findings focused on errors and misconceptions as mentioned in the questions. The review of literature and the theoretical framework helped to elucidate the errors and misconceptions displayed by grade 9 learners. The discussion was classified according to the questions and learners' responses to the tasks as fractions in a context, fractions with algebraic numerators and fractions with algebraic denominators.

\subsection{Fractions in a context}

Questions that we referred to as fractions in a context were those that made learners use chocolate bars to share equally to five friends. Learners seemed to make errors when responding independently to these questions and would realise their mistakes when probed during the interviews. Looking at a response by Learner $A$, the question was as follows: One chocolate bar is shared among 5 friends. How much of the chocolate bar does each friend get?

\subsubsection{Learner A response}

One chocolate bar is shared among 5 friends. How much of the chocolate bar does each friend get? 4 . Use the bar below to show your answer. $20 \div 4=5$ or $5 \times 4=20$

This learner confused the algorithm of fractions as sharing with other operations of multiplication and division. Lukhele et al. (1999) point out that learners perform procedures of numbers in fractions incorrectly and fail to realize that it does not apply to the addition of fractions. Watanabe (2002) identified in his study that representations give meaning to those that created them. In the context of this question, we gave learners the test to write instead of scaffolding them to resolve these questions. The learners were not given chocolate bars to share among 5 friends thus they lost the connection between the real chocolate bars and the representation of a block. Instead of dividing the block into five and showing that each learner would get one equal piece out of the whole, the learner used multiplication and division to incorrectly show that each learner received 4. Cramer et al. (2008) and Osana and Royea (2011) identified that when learners were properly guided to use representations to add fractions, learners understood how to correctly use representations to solve problems. This implies that the learners in this study, like learner A, were not properly guided to use the representation of the block that was to be used to represent a chocolate bar. Bush and Karp (2013) argued that learners have a deficiency 
of simultaneously reasoning abstractly and quantitatively which is shown by this learner when confusing fractions as sharing and operations involved and this was attributed to the lack of conceptual knowledge on fractions.

\subsection{Fractions with Algebraic numerators}

Learners responded to questions on the addition of fractions that had algebraic numerators and numeric denominators, and these were the errors shown;

$$
\text { e.g. } \frac{x}{2}+\frac{x}{3}=\frac{x^{2}}{5}
$$

This learner multiplied the numerators and added the denominators. According to Nesher (1987) this is a display of a misconception where the learner erroneously used already learned concepts of algebra and numerals. We can also say that the learner failed to apply algorithms for fractions which is the use of the LCD. This example shows a misconception on the numerator (multiplying instead of adding) and an error of adding different denominators which leads to a wrong LCD (an error leading to a misconception). The study by Olive (1998) revealed that during the addition of fractions learners had the tendency of adding denominators and numerators which is an erroneous application of already learned concepts of numbers and algebra. There is another addition problem that was erroneously done by learners in their response to the test, and similar to the one above is: $\frac{x}{3}+\frac{1}{4}=\frac{1 x}{7}$

In this example the learner also multiplied the numerator and added the denominators. Therefore, in the context of this study we can conclude that when adding fractions with algebraic numerators, learners multiplied numerators and added denominators. On the other hand, this example illustrates a misconception regarding addition of fractions learners may have developed and teachers need to assist learners to develop acceptable concepts.

\subsection{Fractions with algebraic denominators}

When denominators were variables, some errors and misconceptions were identified from learners' work. These came as a result of learners not finding the LCD; instead they chose to multiply denominators. e.g. $\frac{1}{x y}+\frac{3}{y}=\frac{2 y+3 x y}{2 x y^{2}}$

In this example, the learner erroneously multiplied the denominators and assumed that this was the LCD, and used this to correctly add the fractions. In the study by Olive (1998) and Ramful (2014), some of the findings were that learners multiplied denominators and assumed that it was the LCD. Olive noted that this was not wrong if learners realized that they must simplify their answers.

Another learners' response was this one below where the learner wrongly cancelled a denominator and the numerator, and proceeded with addition of the remaining variables. e.g. $\frac{x+x+x+x+x+x}{x}=5 x$. During the interview, learner A cancelled incorrectly in order to easily solve this problem. According to Watanabe (1991) these are 'short-cuts that learners perform to easily add fractions which lead to errors and misconceptions. This was a misconception of applying addition of natural numbers instead of fractions. The learner did not realize that they must have a single term before dividing through which was wrong as in this learners' example below;

$\frac{x+x+x+x+x+x}{f}=x \frac{(1+1+1+1+1+1)}{x}=6$

\subsection{Fractions with generic denominators}

By generic denominators we mean fractions where denominators had a mixture of numerals and variables. Learners often added the numerators or multiplied them and also multiplied denominators. e.g. 5

b) $\frac{1}{x y}+\frac{4}{2 y}=\frac{5}{2 x y^{2}}$

Ramful (2014) stresses the need for learners to have skills of 'reversibility' and this skill could have assisted learners to avoid errors shown above. The correct answer for $5 b)$ is $\frac{1}{x y}+\frac{4}{2 y}=\frac{2+4 x}{2 x y}$. Learners here could use the rule of the equal sign to identify reversibility together with the procedure of the LCD which results in the problem demanding multiple meanings and representation, and this is lack of conceptual knowledge.

In general we can ascertain that learners exhibited errors and misconceptions because they chose to do 'shortcuts' which did not lead them to the correct answers. We also observed that in most cases learners exhibited errors and misconceptions not necessarily because they were incorrectly taught, but the learners had incorrect conceptions that resulted from the use earlier learned algorithms of natural numbers. We can conclude that learners used "short-cut" 
methods and procedures from their previous knowledge to solve complicated mathematical problems which required different manipulations of algorithms.

\section{References}

Ausubel, D. (1985). Learning as constructing meaning. In Entwistle, N (Eds.). New Direction in Educational Psychology 1: Learning and Teaching. The Falmer Press, London.

Booth, J.L., Newton, K.J. and Twiss-Garrity, L.K. (2014). The impact of fraction magnitude knowledge on algebra performance and learning. Journal of Child Psychology, 118, 110-118.

Bruner, J. (1996). The Culture of Education, Cambridge, MA: Harvard University Press.

Bush, S.B. and Karp, K.S. (2013). Prerequisite algebra and associated misconceptions of middle grade students: A review. The Journal of Mathematical Behavior, 32, 613-632.

Davydov, V. (1995). The Influence of L.S. Vygotsky on Education Theory, Research, and Practice. Educational Researcher, 4(3), 12-21.

Dunzenli-Gokalp, N. and Sharma, M.D. (2010). A study on the addition and subtraction of fractions: The use of Pirie and Kieren model and hands on activities. Procedia Social and Behavioral Sciences, 2, 5168-5171.

Ellerton, N. (1991). Classroom Discourse and Mathematics Learning. Proceedings of the $15^{\text {th }}$ annual conference of the International Group for the Psychology of Mathematics Education, 2, 17-24.

Cramer, K., Wyberg, T., and Leavitt, S. (2008). The Role of Representations in Fraction Addition and Subtraction. The National Council of Teachers of Mathematics. www.nctm.org. Accessed January 20, 2014.

Gabriel, F., Coche, F., Szucs, D., Carette, V., Rey, B. and Content, A. (2013). A component view of children's difficulties in learning fractions. Frontiers in Psychology, 4, Doi:10.3389/fpsyg.2013.00715 Accessed January 20, 2014.

Hallett, D., Nunes, T., Bryant, P. and Thorpe, C.M. (2012). Individual differences in conceptual and procedural fraction understanding: The role of abilities and school experience. Journal of Experimental Child Psychology, 113, 469-486.

Hart, K. (1981). In children's understanding of mathematics. In Hart, K.M. et al. (Ed.). John Murray Publishers, London, 11-16.

Hecht, S.A. and Vagi, K.J. (2012). Patterns in children's knowledge about fractions. Journal of Experimental Child Psychology, 111, 212 229.

Idris, N. (2011). Error Patterns in Addition and Subtraction of Fractions among Form Two Students. Journal of Mathematics Education, $4(2), 35-54$.

Kong, S.C. (2008). The development of a cognitive tool for teaching and learning fractions in the mathematics classroom: A designbased study. Computers and Education, 51, 886-899.

Lukhele, R.B., Murray, H., and Olivier, A. (1999). Learners' understanding of the addition of fractions. Proceedings of the Fifth Annual Congress for Mathematics Education of South Africa, 1, 87-97.

Luneta, K. and Makonye, P. (2010). Learner errors and misconceptions in elementary analysis: A case study of a grade 12 class in South Africa. Acta Didactica Napocensia, 3(3), 35-45.

Norton, A. and Boyce, S. (2013). A cognitive core for common state Standards. The Journal of Mathematical Behavior, 32, 266-279.

Nesher, P. (1987). Towards an Instructional Theory: the role of Student's Misconceptions. For the Learning of Mathematics, 7, 33-39.

Olive, J. (1998). Modifications of children's multiplicative operations with whole numbers and fractions that may generate rational numbers of arithmetic. Proceedings of the $18^{\text {th }}$ PME-NA conference, 316-323.

Olivier, A. (1989). Handling pupils' misconceptions. Pythagoras, 21, 10-19.

Ramful, A. (2014). Reversible reasoning in fractional situations. Theorems-in-action and constrains. The Journal of Mathematics Behavior, 33, 119-130.

Siegler, R.S., Thompson, C, A. and Schneider, M. (2011). An Integrated theory of whole number and fractions development. Cognitive Psychology.doi:10.1016/j.cogpsych.2011.03.001

Siegler, R.S., Fazio, L.K., Bailey, D.H. and Zhou, X. (2013). Fractions: the new frontier for theories of numerical development. Trends in Cognitive Sciences, 17(1), 13-19. ｈttp://dx.doi.org/10.1016/j.tics.2012.11.004 Accessed January 20, 2014.

Osana, H.P. and Royea, D.A. (2011). Obstacles and challenges in pre-service teachers' exploration with fractions: A view from a smallscale intervention study. The Journal of Mathematics Behavior, 30, 333-352.

Watanabe, T. (2002). Representations in Teaching and Learning Fractions. The National Council of Teachers of Mathematics. www.nctm.org. Accessed January 10, 2014.

Watanabe, T. (1995). Preservation of the common referent in the Addition of Fractions: A case study PME-NA, 17, 390-395.

Watanabe, T. (1991). Incongruity of the common referent in the Addition of Fractions: A case study of simple fractions, PME-NA, 17, 390-395.

Watts, W. and Bentley, D. (1991). Constructivism in the curriculum: Can we close the gap between the strong theoretical version and the weak version of theory in practice. The Curriculum Journal, 2(2), 171-182.

Vygotsky, L.S. (1978). Mind in Society. Massachusetts: Harvard University Press. 